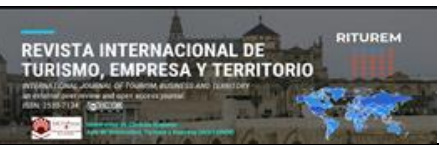

Cita bibliográfica: Tortul, M., Elías, S. y Leonardi, V. (2020). Los Parques Nacionales Argentinos declarados Patrimonio de la Humanidad. Un análisis de su atractividad turística. Revista Internacional de Turismo, Empresa y Territorio, 4 (2), 36-51. https://doi.org/10.21071/riturem.v4i2.13027

\title{
Los Parques Nacionales Argentinos declarados Patrimonio de la Humanidad. Un análisis de su atractividad turística ${ }^{1}$
}

\section{Argentine National Parks nominated as World Heritage Sites. An analysis of their tourist attractiveness}

\author{
Marina Tortul
}

Silvina Elías ${ }^{2}$

Viviana Leonardi ${ }^{3}$

\section{Resumen}

Desde el año 1972, la UNESCO incorpora en su lista de Patrimonio las áreas geográficas que por sus características culturales y/o naturales son objeto de cuidado y preservación. Cuando un Parque Nacional es declarado sitio Patrimonio Mundial (SPM) aumenta su jerarquización en la conservación de la biodiversidad y presumiblemente el flujo de turistas. En Argentina, hay declarados 13 SPM, de los cuales 4 constituyen Parques Nacionales (UNESCO, 2019). El objetivo del presente trabajo es identificar si el arribo de turistas a Parques Nacionales Argentinos (PN) es mayor en aquellos que reciben una nominación UNESCO como SPM. La metodología empleada ha sido descriptiva y se ha trabajado con información secundaria de la Dirección de Parques Nacionales y del Anuario Estadístico de Turismo. El análisis descriptivo estudia la distribución de los parques en el territorio argentino, el arribo de turistas a los mismos, su concentración y crecimiento durante el período 1991-2015. Los resultados muestran que el turismo de PN ha crecido exponencialmente durante 1991-2015, observándose una marcada preferencia por los PN declarados SPM (Iguazú, Talampaya, Los Alerces y Los Glaciares).

Palabras clave: Parques Nacionales, Sitio Patrimonio Mundial, Turismo, Argentina.

\footnotetext{
${ }^{1}$ Este trabajo se realizó en el marco del Proyecto de Investigación "Análisis económico de la cultura y el turismo cultural", Universidad Nacional del Sur, Bahía Blanca, Argentina. Una versión previa del mismo fue presentada en el Congreso Internacional de Investigaciones en Turismo e Identidad: aportes para construcción de una mirada interdisciplinaria y científica. Universidad Nacional de Mendoza. Mendoza, Argentina. 26 y 27 de septiembre de 2019.

*Autor para la correspondencia. Departamento de Economía, UNS. IIESS, UNS-CONICET, Argentina. Email: marina.tortul@uns.edu.ar Id Orcid: https://orcid.org/0000-0002-8739-6049

${ }^{2}$ Departamento de Economía, UNS. IIESS, UNS-CONICET, Argentina. Email: slias @uns.edu.ar. Id. Orcid: https://orcid.org/0000-0001-7750-1821

${ }^{3}$ Departamento de Economía, UNS. IIESS, UNS-CONICET, Argentina. Email: viviana.leonardi@uns.edu.ar .

Id. Orcid: https://orcid.org/0000-0002-4289-5039
} 


\begin{abstract}
Since 1972, UNESCO has included in its Heritage list geographical areas that, due to their cultural and/or natural characteristics, are preserved. When a National Park is declared a World Heritage Site (WHS), its hierarchy in the conservation of biodiversity and, presumably, the flow of tourists increases. In Argentina, there are 13 WHS declared, and four constitute National Parks (UNESCO, 2019). Thus, the aim of this research is to study whether the arrival of tourists is higher in those Argentine National Parks (NP) nominated as WHS. The methodology is descriptive, working with secondary information from the National Parks Directorate and the Tourism Statistical Yearbook. The descriptive analysis studies the distribution of the parks in the Argentine territory, the arrival of tourists, their concentration and growth during the period 1991-2015. The results show that tourism in NP has grown exponentially during the period analyzed, with a marked preference for the NP declared WHS (Iguazú, Talampaya, Los Alerces and Los Glaciares).
\end{abstract}

Keywords: National Parks, World Heritage Site, Tourism, Argentina.

\title{
1. Introducción
}

Tanto en el plano nacional como internacional, el turismo es una de las actividades humanas asociada estrechamente al uso de áreas naturales protegidas. La mayoría de los atractivos de alta jerarquía no creados por el hombre están localizados en estas áreas naturales especiales con el propósito de su conservación para las futuras generaciones. Así, la relación entre el turismo y la inscripción de un lugar como Patrimonio de la Humanidad se está convirtiendo en un aspecto más para analizar en la literatura científica sobre patrimonio y turismo cultural.

La "Convención sobre la Protección del Patrimonio Natural y Cultural” celebrada en 1972 por la Organización de las Naciones Unidas para la Educación, la Ciencia y la Cultura significó la puesta en marcha de un programa de reconocimiento de áreas naturales protegidas. El objetivo básico de la Convención de 1972 es la corresponsabilidad internacional en la preservación de bienes patrimoniales con valor excepcional universal. (UNESCO, 2000).

En Argentina el Sistema Nacional de Áreas Protegidas (SNAP) cuenta, en cada región del país, con áreas que conservan muestras representativas de la inmensa biodiversidad del territorio. La Administración de Áreas Protegidas tiene como objetivo diseñar, conducir y controlar la ejecución de las políticas necesarias para conservar y manejar las diferentes áreas de conservación. Este último es un organismo descentralizado dependiente de la Secretaria de Turismo del Ministerio de Turismo de la Nación. Las clasificaciones de áreas protegidas en la Argentina son: Reserva Natural Educativa, Reserva de la Defensa, Reserva Natural, Reserva Nacional, Parque Nacional, Monumento Natural, Área Marina Protegida, Reserva Natural Estricta y Parque Interjurisdiccional Marino. Cuatro Parques Nacionales Argentinos han sido declarados Sitio Patrimonio Mundial (SPM) cuyas nominaciones se han en dado diversos años: Parque Nacional Los Glaciares, en 1981; el Parque Nacional Iguazú, en 1984; el Parque Nacional Talampaya, en el año 2000 y el Parque Nacional Los Alerces, en el 2017. Según un informe de la Consultora Elypsis (2018), durante el año 2018 casi 4 millones de turistas visitaron los parques nacionales y el turismo en general movilizó 15.000 millones de dólares, de los cuales el 30\% fue generado por el turismo de naturaleza. Entre 1990 y 2017, las visitas se multiplicaron por cinco (711 mil personas a 3,8 millones) y se duplicaron desde 2001 . De acuerdo con el estudio, este tipo de turismo podría crecer en 6400 millones de dólares en los próximos 10 años y generar 300.000 puestos de empleo. 
En este contexto, el objetivo de este trabajo es identificar si el arribo de turistas a Parques Nacionales Argentinos (PN) es mayor en aquellos que reciben una nominación UNESCO como SPM. Para ello se estudia la evolución del arribo de turistas a parques nacionales argentinos, distinguiendo entre aquellos declarados SPM y aquellos no SPM, a partir de información secundaria de la Dirección de Parques Nacionales Argentinos y del Anuario Estadístico de Turismo (AET, 2007-2015).

El resto del trabajo se organiza como sigue. La sección siguiente constituye el marco de referencia teórico, en la que se revisan algunos antecedentes sobre la relación entre el turismo y los SPM y la política argentina de parques nacionales. En la tercera sección se explicita la metodología del trabajo. En la cuarta, se exponen los resultados y finalmente se obtienen diversas conclusiones.

\section{Marco de referencia}

\subsection{Sitios Patrimonio Mundial y Turismo}

La Lista de Patrimonio Mundial es el registro de bienes patrimoniales de mayor reconocimiento y alcance planetario, muchos de los cuales tienen una proyección espacial compleja, por lo que este registro se convierte en un interesante laboratorio para la identificación, protección y gestión de los paisajes patrimoniales. La Lista identifica específicamente estos paisajes (paisajes culturales), pero también reconoce otros bienes que comparten esa proyección espacial compleja; especialmente los llamados bienes mixtos. Para Silva Pérez y Fernández Salinas (2015), la Lista es hoy, antes que nada, un registro prestigioso en los ámbitos académicos, políticos y ciudadanos. Estos autores consideran que tras varios decenios de gestión, de la Lista pueden inferirse numerosos aspectos positivos: corresponsabilidad internacional en la defensa del patrimonio; aportación de un corpus conceptual y metodológico; reforzamiento de la visibilidad del patrimonio y de sus posibilidades para el desarrollo socioeconómico, etcétera. Entre aquel año y la actualidad, la creación de figuras tales como "sitios de patrimonio de la humanidad", "sitios hemisféricos", "Sitios Ramsar" y "Reservas de la Biosfera", otorga a cada nación miembro el derecho a recibir cooperación técnica y económico-financiera y exige el deber de cumplimentar con las normas conservacionistas promovidas para tales fines (UNESCO 2000).

Se encuentran inscritos en la lista del Patrimonio Mundial de la UNESCO (2019) un total de 1.073 bienes (832 culturales, 206 naturales y 35 mixtos) en 167 estados. Adicionalmente, 1.629 bienes están intentando formar parte de esta lista, muchas veces motivados, además de la conservación, por la posibilidad del incremento de flujos turísticos capaces de generar una serie de impactos positivos en el territorio; siendo éste uno de los principales motivos que inspiraron la creación de la Convención del Patrimonio Mundial en 1972. Actualmente, 190 países han ratificado esta convención y forman parte de una comunidad internacional unida en la misión conjunta de identificar y proteger el patrimonio natural y cultural más importante del planeta (UNESCO, 2019).

Ashworth y Turnbridge (1990), Pocock (1997) y Shackley (1998), entre otros, encuentran que el impacto económico real de la inscripción en la Lista de Patrimonio de la Humanidad como el crecimiento de turistas son positivos. Galvin (1997) muestra que la visita a parques patrimonio en Estados Unidos fueron 5,2\% superior que las visitas a los parques nacionales sin el título de patrimonio UNESCO durante el período 1990-1995. Sin embargo, estudios empíricos realizados a partir del 2000 han arrojado resultados diversos. Algunos autores 
plantean que el nexo entre el listado de SPM y la cantidad de visitantes es débil, más allá de las tendencias de turismo, especialmente para los sitios que fueron importantes puntos de interés antes de la lista del patrimonio. De este modo, no existe un consenso acerca de la efectividad en un sentido turístico de este reconocimiento por parte de la UNESCO (Elías, Leonardi y Tortul, 2018), como incluso tampoco sobre la declaración oficial como Parques Naturales de otros tipos de espacios naturales protegidos (Mulero y Rivera, 2018).

\subsection{Parques nacionales y sitios Patrimonio en Argentina}

En Argentina, la enunciación de la temática conservacionista estuvo situada alrededor de los proyectos Nahuel Huapi e Iguazú. Estas propuestas, acuñadas en los albores del siglo XX, fueron protagonizadas por hombres que gozaban de marcado prestigio entre sus contemporáneos. En tanto que la idea de creación del Parque Nacional Nahuel Huapi fue enunciada por Francisco Pascasio Moreno, naturalista y explorador de estrecha vinculación con ámbitos institucionales relacionados al dominio territorial, y continuada por Bailey Willis, ingeniero y geólogo norteamericano contratado por el gobierno nacional para la ejecución de obras de infraestructura tendientes al desarrollo de la región patagónica, el proyecto del Parque Nacional Iguazú estuvo originado en una iniciativa gubernamental que, con igual propósito para la región noreste del país, encomendó su diseño técnico a Charles Thays, arquitecto y paisajista de origen francés radicado en nuestro país. Ambos proyectos fundacionales de áreas naturales protegidas reconocen a la experiencia norteamericana como su principal fuente de inspiración (Benjamín y Gutiérrez 1985).

Para Melina Piglia (2012) la política de parques nacionales fue el primer ensayo consistente y sistemático de política turística nacional. Desde las décadas finales del siglo XIX, una serie de discursos científicos, literarios y políticos cifraron la identidad nacional argentina en las bellezas y riquezas "naturales" del territorio. En octubre de 1934 se sancionó la ley de Parques Nacionales, que creaba la DPN, dependiente del Ministerio de Agricultura. Ezequiel Bustillo, un abogado conservador con una breve trayectoria relacionada con el Parque del Sur, la presidió desde su creación hasta 1944, en una gestión con fuerte sello personal: la política de Parques fue, en buena medida, fruto de sus ideas y de su red de contactos personales. La autora sostiene que el fomento y la organización del turismo en los parques nacionales, ocupó un lugar central en la política de Bustillo. Su proyecto tenía dos objetivos principales: de un lado, el desarrollo económico de las zonas comprendidas en los parques, desde la perspectiva de su contribución a la riqueza de la nación, y, del otro, su integración simbólica y material al territorio nacional. El turismo era, a los ojos de Bustillo el instrumento clave para realizar simultáneamente los dos objetivos, a partir de la transformación de la región en un centro de "gran" turismo (turismo de elite e internacional), que luego podría dar lugar a un desarrollo turístico más amplio. El turismo permitiría el encuentro de la elite cosmopolita con los sublimes paisajes emblemáticos de la nación. Por otro lado, era también una industria y podría traer prosperidad y ayudar al desarrollo económico y al poblamiento.

La política turística de Parques se abocó a la construcción de los parques nacionales como lugares turísticos, a través de la remodelación del paisaje, de la inversión en infraestructura y de su instalación en el imaginario como lugar turístico deseable y como paisaje patriótico. Se basaba en la propiedad estatal de los atractivos turísticos y podía imponer por lo tanto en su jurisdicción, una estrecha supervisión de la actividad privada en todo lo que pudiera afectar al desarrollo del turismo en el sentido en que se lo había planificado; había un elemento de regulación del recurso turístico y de la oferta, a través, no solo del control de los servicios 
turísticos, sino incluso de la propia urbanización y de todas las acciones que pudieran afectar al paisaje (Piglia, 2012).

Según la Administración de Parques Nacionales, Argentina cuenta con un sistema de 38 parques nacionales destinado a proteger el patrimonio natural y cultural del país que ocupa casi un $4 \%$ del territorio (3,8 millones de hectáreas, cuidados por aproximadamente unos 200 guardaparques). Cuatro de estos parques son Sitio Patrimonio Mundial (SPM) cuyas nominaciones se han en dado diversos años: Parque Nacional Los Glaciares, en 1981; el Parque Nacional Iguazú, en 1984; el Parque Nacional Talampaya, en el año 2000 y el Parque Nacional Los Alerces, en el 2017. De esta manera, se jerarquizan los niveles de conservación de la biodiversidad y promueve un mayor número de visitantes en función de la trascendencia mundial de esta distinción.

\section{Metodología y herramientas de trabajo}

Con el fin de alcanzar el objetivo propuesto, se ha trabajado básicamente con información secundaria de la Dirección de Parques Nacionales y del Anuario Estadístico de Turismo y la explotación específica de su Banco de Datos. Se ha estudiado, asimismo, la distribución de los parques en el territorio argentino diferenciando aquellos que han obtenido la nominación de SPM.

Seguidamente, se ha analizado el volumen de la frecuentación turístico-recreativa de visitantes a los Parques Nacionales argentinos en el período1991-2015, observando si existe preferencia por los parques declarados SPM. Dicho análisis se realiza utilizando el método de estadística descriptiva, el cual permite explorar los datos a fin de identificar sus principales características mediante un número reducido de gráficos y/o números. En este análisis se consideraron las siguientes variables: (i) arribo de turistas según país de origen, (ii) análisis del arribo de turistas a Parques Nacionales según país de origen, (iii) análisis del arribo de turistas a Parques Nacionales según área geográfica. Para analizar los cambios estructurales de tendencia en el arribo de turistas a Parques Nacionales se ha aplicado el test de Zivot y Andrews (1992).

\section{Resultados y Discusión}

\subsection{Parques Nacionales en Argentina}

Argentina cuenta con 40 Áreas Protegidas Nacionales, incluyendo 29 Parques Nacionales, que ocupan 3.870.261 hectáreas (AET, 2015). El 97\% de la superficie protegida está constituida específicamente por Parques Nacionales (PN) distribuidos heterogéneamente a lo largo del país. Con 11 PN, la región patagónica concentra la mayor extensión de PN del país, le siguen la región norteña con 8 PN, la región cuyana con 3 PN, la región litoraleña con 6 PN y la región central con solo 1 PN (Figura 1). 
Figura 1: Distribución de los parques nacionales en el territorio argentino

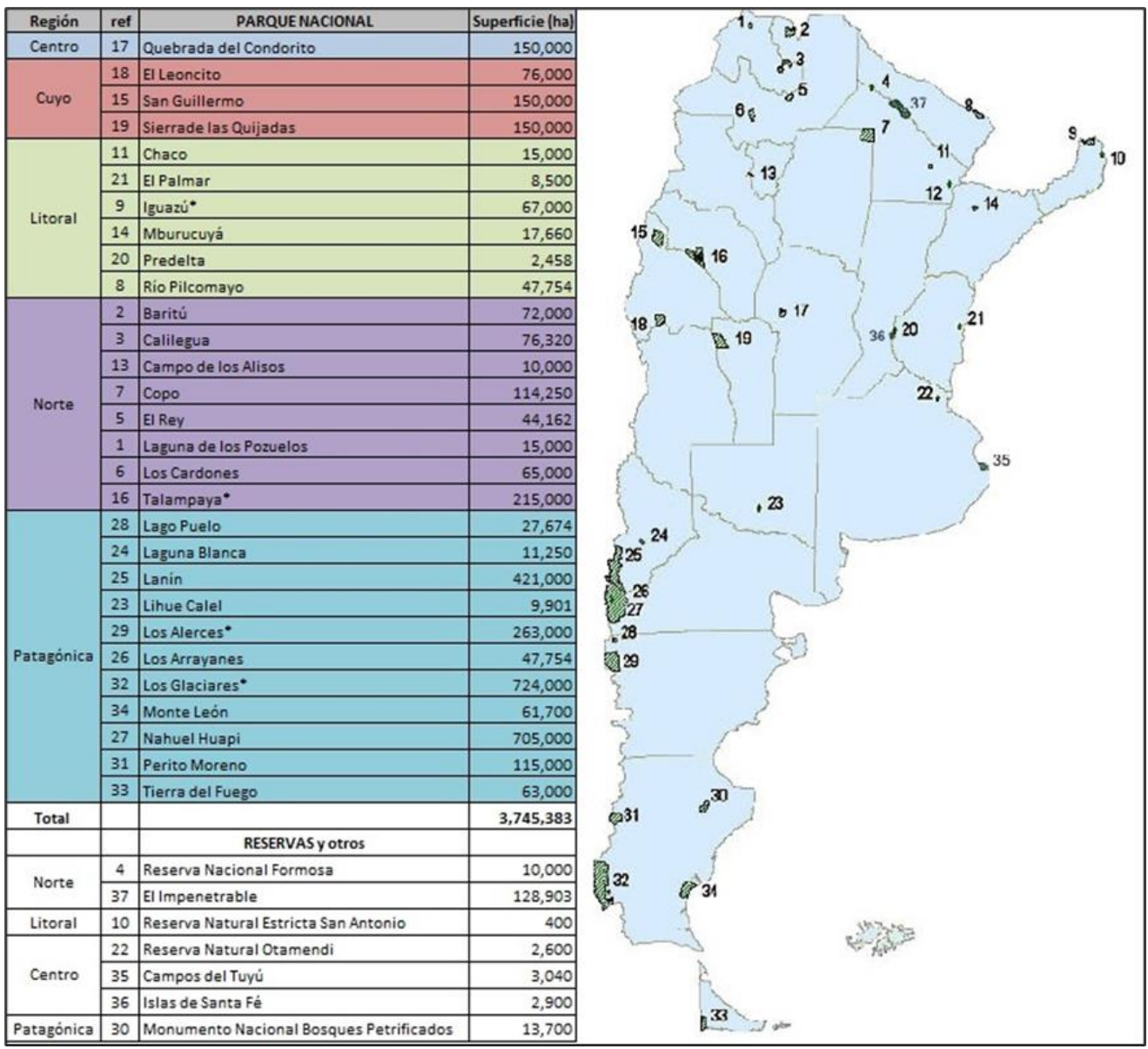

Fuente. ARGENTUR, 2016. Referencias de edición propia.

Cuatro de estos parques son Sitios Patrimonio Mundial (SPM) cuyas nominaciones se han dado en diversos años: Parque Nacional Los Glaciares, en 1981; el Parque Nacional Iguazú, en 1984; el Parque Nacional Talampaya, en el año 2000 y el Parque Nacional Los Alerces, en el 2017. A continuación, se presenta una breve descripción de cada uno de ellos:

$\checkmark$ Parque Nacional Los Glaciares: el glaciar Perito Moreno es una inmensa masa deslizante de hielo que se descuelga desde Los Andes hacia el lago Argentino, formando una muralla gigantesca de hielo natural de 4 kilómetros de frente y 60 metros de altura sobre el nivel del lago. El Perito Moreno, junto a doce glaciares más, conforman el Parque Nacional Los Glaciares. Este parque se ubica en el sudoeste de la provincia de Santa Cruz, posee una superficie de 726.927 hectáreas y fue creado para preservar una extensa área de hielos continentales y glaciares, del bosque andino-patagónico austral y muestras de la estepa patagónica. Es el más extenso del Sistema Nacional de Áreas Protegidas Argentinas. En cualquier época del año asombran sus frecuentes derrumbes de torres de hielo que se fragmentan en múltiples témpanos flotantes. Este imponente espacio natural es el primer sitio de Argentina que fue incluido por la UNESCO en su lista de Patrimonio Mundial en el año 1981, cuando declaró como tal al Parque Nacional Los Glaciares, en la provincia de Santa Cruz. En la actualidad, el glaciar Perito Moreno es uno de los pocos glaciares en el mundo que sigue avanzando e incrementando su volumen (Figura 2). 
Parque Nacional Iguazú: hacia el extremo nordeste del país, concretamente en la provincia de Misiones, se encuentra este espectáculo natural compuesto por un imponente conjunto de 275 cascadas, extendidas a lo largo de $2.7 \mathrm{Km}$. Posee una superficie de 67.620 hectáreas pertenecientes a la ecorregión Selva Paranaense. El río Iguazú, que significa en guaraní agua grande, desemboca en el Paraná y corre con una anchura de 1.500 metros, salpicando islas e islotes para desembocar en un barranco de lava formado hace 120 millones de años. El Parque Nacional Iguazú fue designado Patrimonio Mundial de la Humanidad por la UNESCO en 1984. Además, las Cataratas del Iguazú, una de las nuevas Maravillas del Mundo (Figura 3).

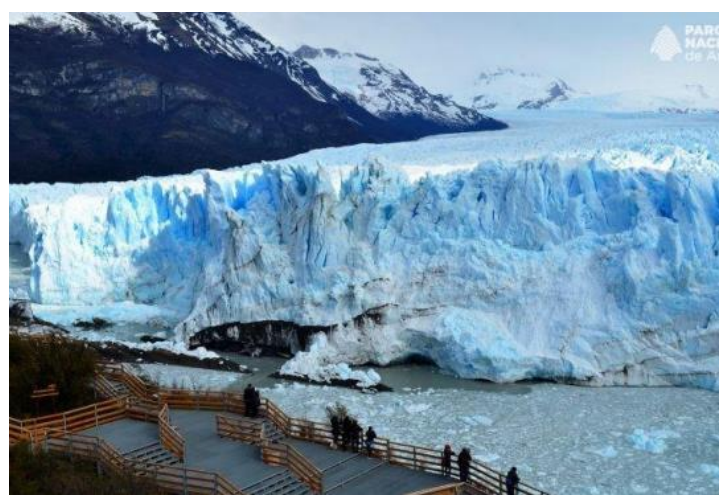

Fuente: Fotografía de Parques Nacionales de Argentina

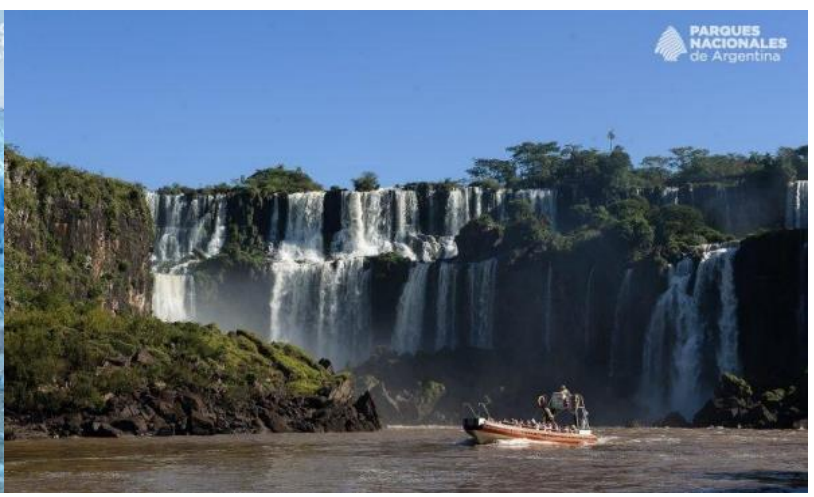

Fuente: Fotografía de Parques Nacionales de Argentina

$\checkmark$ Parque Nacional Talampaya: está ubicado sobre el centro oeste de la provincia de La Rioja, en proximidad (60 km) de Villa Unión y fue creado el 10 de julio de 1997, por ley 24.846. Posee una superficie de 213.800 hectáreas pertenecientes a la ecorregión Monte de Sierras y Bolsones. Esta área también comprende un sector de la provincia de San Juan. En el año 2000 la UNESCO declaró Sitio de Patrimonio Mundial, el área compuesta por el Parque Nacional Talampaya y el Parque Provincial Ischigualasto, denominándolos Parques Naturales Ischigualasto / Talampaya (Figura 4).

$\checkmark$ Parque Nacional Los Alerces: está ubicado en el oeste de la provincia de Chubut. Posee una superficie de 259.570 hectáreas pertenecientes a la ecorregión de Bosques Patagónicos. El Decreto No 105.433 de 1937 fijó para distintos territorios en la Patagonia andina la condición de reservas con destino a la posterior creación de Parques Nacionales. El parque se halla emplazado en un valle glaciario principal que vincula a otros que convergen en él, constituyendo una red de lagos: Lago Futalaufquen, Lago Verde, Lago Rivadavia, Lago Menéndez, Lago Cisne, etc. El 7 de julio de 2017 la UNESCO declaró Sitio de Patrimonio Mundial a 188.379 hectáreas del Parque Nacional Los Alerces, de las cuales más de siete mil protegen Bosques Milenarios de Alerces, con ejemplares que alcanzan 2.600 años de existencia (Figura 5). 
Figura 4. Parque Nacional Talampaya

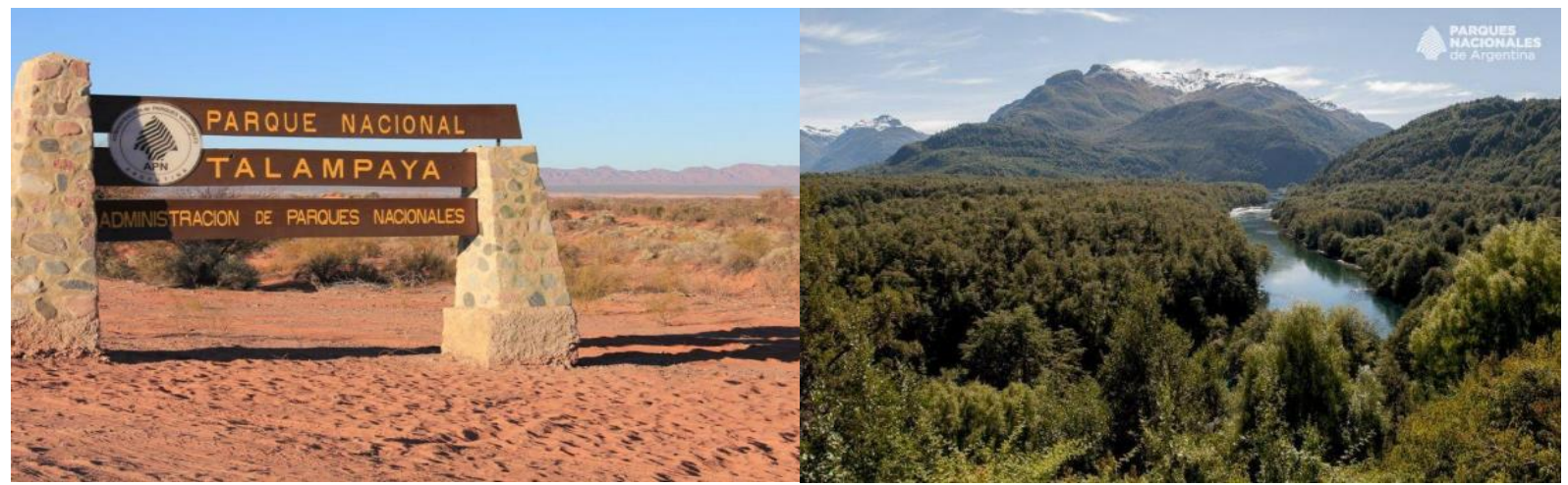

Fuente: Fotografía de Parques Nacionales de Argentina
Figura 5. Parque Nacional Los Alerces

Fuente: Fotografía de Parques Nacionales de Argentina

\subsection{Arribo de turistas a Parques Nacionales Argentinos}

\subsubsection{Un análisis para el total del país}

De acuerdo con los datos del Ministerio de Turismo (AET, 2007-2015), el turismo de PNA presenta un crecimiento exponencial en el período de 1991-2015 (Figura 6), pasando de poco más de 700.000 de turistas en 1991 a poco más de 3.5 millones en 2015.

El arribo de turistas a PNA presenta una tasa de crecimiento promedio anual del $8 \%$ durante el periodo de análisis. Sin embargo, vale señalar que esta evolución no fue homogénea a nivel territorial.

Por el contrario, se pueden identificar dos subperíodos de características diferentes ${ }^{4}$, probablemente asociados al cambio del modelo de crecimiento socioeconómico del país. El primero, comprendido entre 1991-2001, presenta un crecimiento moderado con una tasa promedio anual del $5 \%$.

En el segundo subperíodo de 1992- 2015 el crecimiento es exponencial con una tasa promedio anual del $9 \%$. En esta etapa se destaca el efecto de la crisis financiera internacional 2008-2009, con una caída de 13\% en el flujo de turistas a PNA.

\footnotetext{
${ }^{4}$ De acuerdo al test de Zivot y Andrews (1992), la serie presenta un quiebra estructural de tendencia muy significativa en el año 2001.
} 
Figura 6. Volumen de turistas en los Parques Nacionales Argentinos 1991-2015

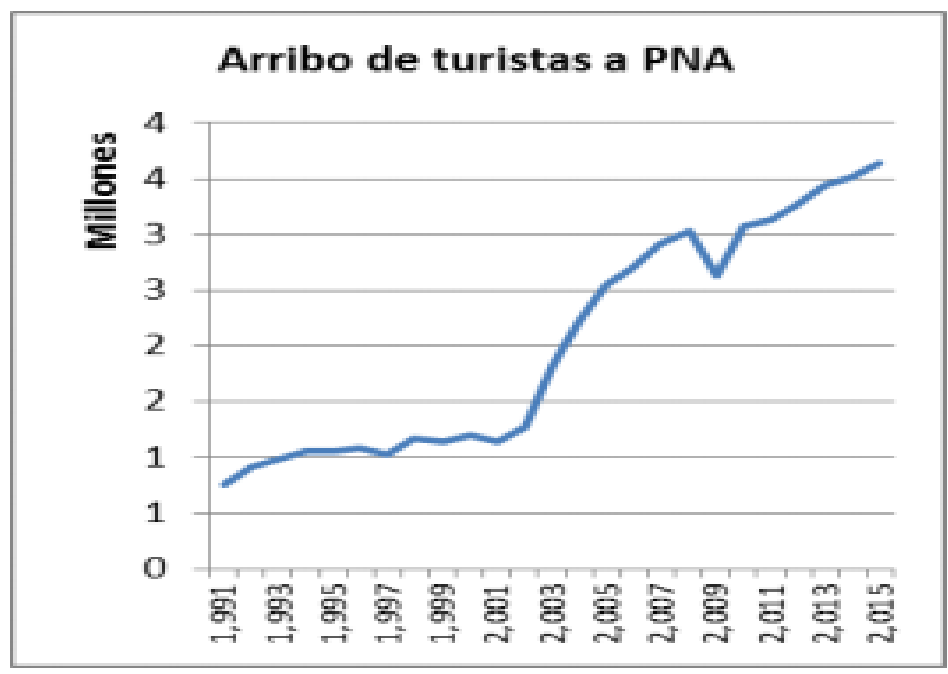

Fuente: Elaboración propia a partir de datos de los Anuarios Estadísticos de Turismo (2007-2015).

Presumiblemente, dicha caída se asocia a la desaceleración del arribo de turistas extranjeros (Figura 7), que pasa de una tasa de crecimiento anual del 60\% entre 2007-2008 a una del 13\% entre 2009-2010. Asimismo, también se observa una importante disminución de la participación de los turistas extranjeros a partir de 2011. En particular, las tasas de crecimiento anuales muestran una desaceleración, pasando de una tasa de crecimiento anual del 17\% entre 2009-2010 a una tasa promedio de 7\% en el periodo 2011-2015. Teniendo en cuenta que, en términos generales, el 12.2\% (AET, 2015) de los turistas no residentes proceden de Europa, posiblemente este comportamiento esté asociado a la crisis de deuda que sufrían los países de Europa en ese entonces.

Figura 7. Importancia del turismo extranjero en los PN argentinos

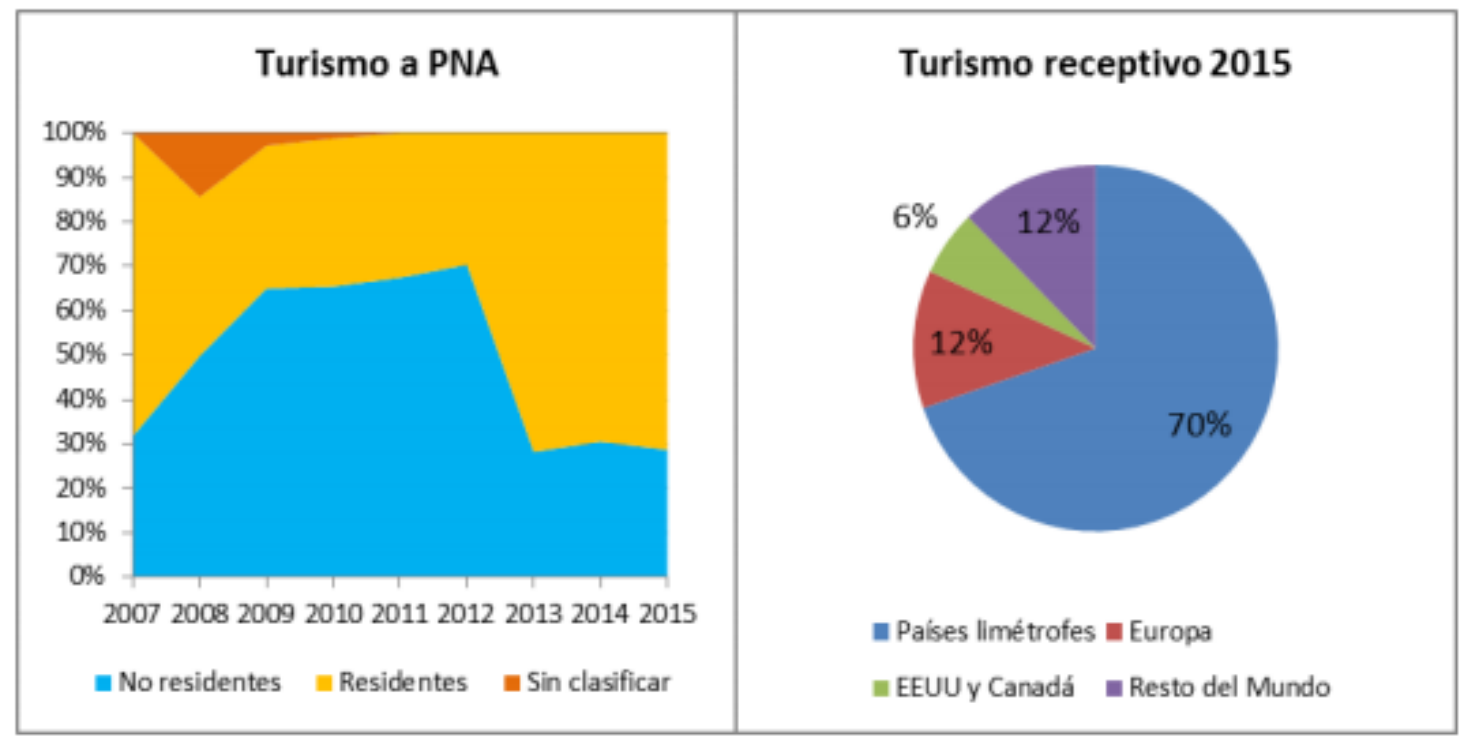

*PN declarados Sitio Patrimonio de la Humanidad (UNESCO, 2019).

Fuente: Elaboración propia a partir de los Anuarios Estadísticos de Turismo (2007-2015). 
A pesar de dicha diversidad, y de acuerdo con los datos del Ministerio de Turismo (AET, 2007-2015), en promedio para el período 1991-2015 (Figura 8), el 70\% del arribo de turistas a los PN argentinos estuvo concentrado en solo tres parques: PN Iguazú (41\%), PN Nahuel Huapi (15\%) y PN Los Glaciares (12\%). Como ya se mencionará, dos de ellos son considerados sitios patrimoniales de acuerdo a la UNESCO (2019).

El turismo en los PNA SPM ha experimentado una evolución creciente pasando del $56 \%$ al inicio del periodo la $63 \%$ al final del mismo. Así pues, se corrobora la preferencia general por los PNA SPM con respecto al resto de los PNA. Sin embargo, a nivel interno dentro de este grupo de Parques Nacionales, el comportamiento es variable. En particular, se verifica una evolución negativa en el PN SPM Iguazú, que pasa del 50\% al 38\%, contrarrestada por la participación creciente del resto de los PNA SPM en la cuota de mercado de turismo: Los Alerces pasan del 1\% al 5\%, Los Glaciares del 5\% al 20\% y Talampaya, que no constituía un sitio de interés al principio del período, alcanza el $2 \%$ al final. Asimismo, se observan cambios de preferencia en el resto de los PNA. Se destaca la pérdida de interés por el PN Nahual Huapi, que pasa de $28 \%$ en 1992 al $9 \%$ en 2015, parcialmente contrarrestada por la participación creciente del resto de los PNA, que pasan de $16 \%$ al $28 \%$, respectivamente.

Figura 8. Arribo de turistas a Parques Nacionales argentinos 1991-2015
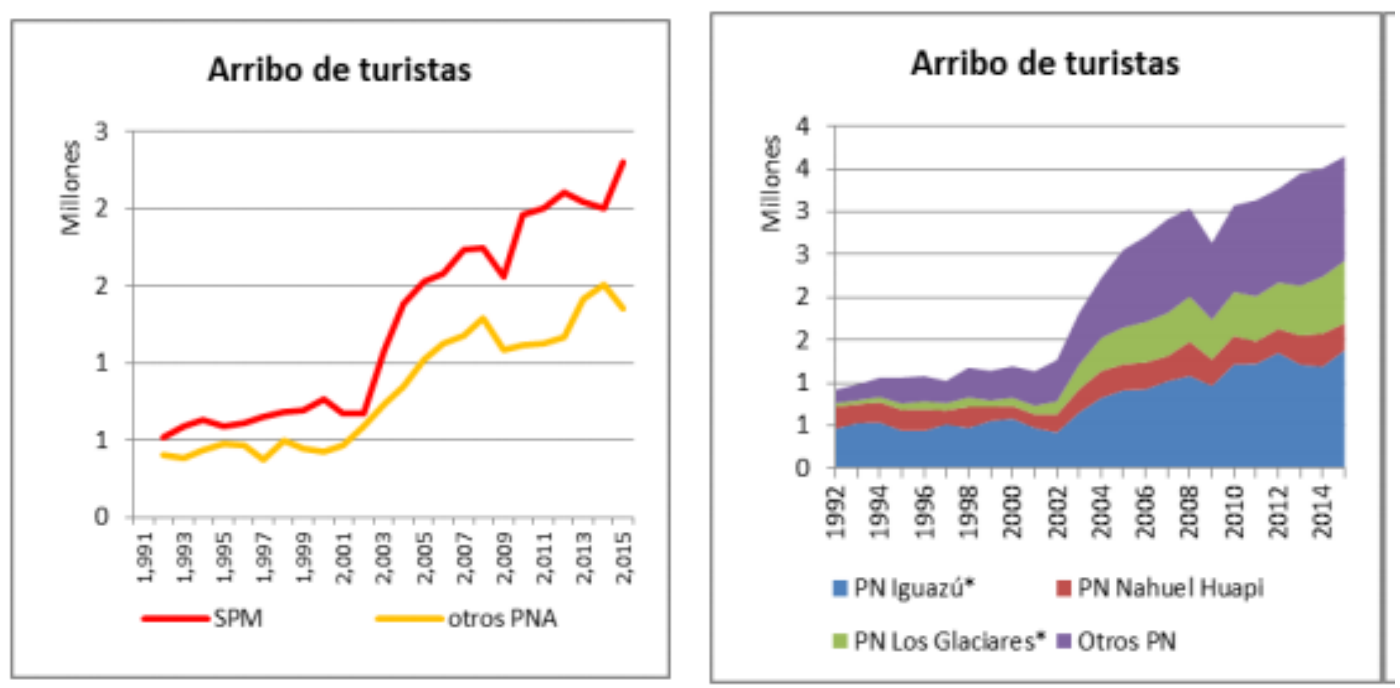

Fuente: Elaboración propia en base a datos de los Anuario Estadísticos de Turismo (2007-2015)

En cuanto al tipo de turistas que arriban a los parques, se observa que, si bien en términos absolutos los PNA SPM reciben más turistas extranjeros que los otros PNA, en términos relativos la situación es diferente (Figura 9). Con un promedio de 2.5 turistas extranjeros por cada turista nacional contra un promedio de 1.1, se observa la supremacía relativa de la exportación de servicios turísticos a PN no declarados Patrimonio de la Humanidad con respecto a los PN UNESCO (2019). Incluso esta relación es relativamente estable entre 20072015 para el caso de este último tipo de PN, mientras que para los primeros oscila entre $0.1 \mathrm{y}$ 5.4 turistas extranjeros por cada turista nacional. Vale notar cómo el indicador se derrumba, y se invierte la relación a partir de 2012. De esta manera, a partir de entonces se observa la mayor presencia de turistas residentes con respecto a los no residentes. En particular, se observa que por cada turista nacional arriban en promedio 0.7 turistas extranjeros a los PN Sitios 
Patrimoniales y 0.1 a los otros PN. Posiblemente esta inflexión en el tipo de turistas que reciben los PN esté asociada al contexto internacional ya mencionado.

Figura 9. Turistas residentes y no residentes

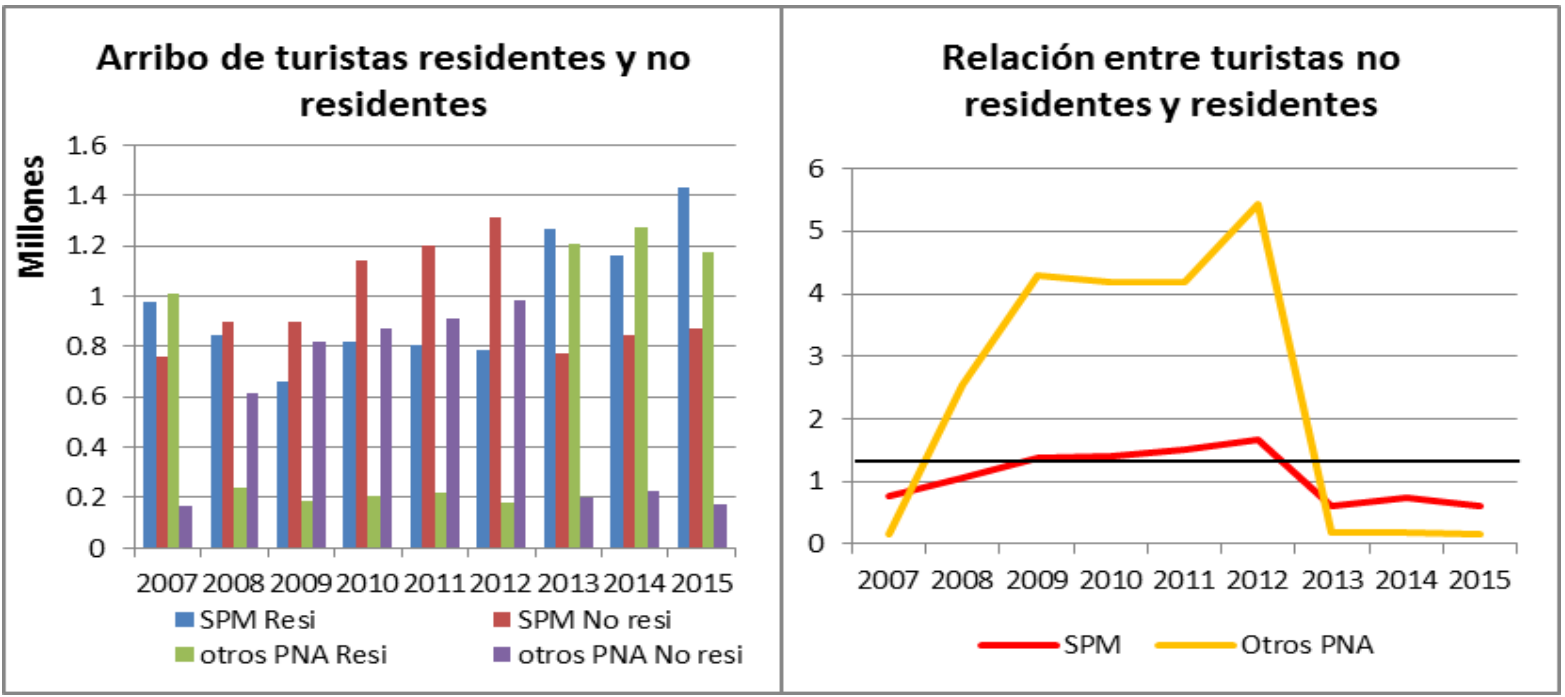

Fuente: Elaboración propia a partir de los Anuarios Estadísticos de Turismo (2007-2015).

Finalmente, teniendo en cuenta la localización geográfica de los diferentes parques, el atractivo turístico de las regiones de PN del país es heterogéneo (Figura 10). Con una participación del $48 \%$ cada una, la Patagonia y el Litoral son las preferidas por los turistas. Le siguen el Norte (3\%), Cuyo (1\%) y el Centro. Vale destacar, sin embargo, que tanto la cantidad de PN como la extensión de los mismos difieren entre regiones. Aun así, no parece existir una relación significativa entre la superficie de los PN y la afluencia de turistas, dado que se observa que las regiones preferidas abarcan superficies muy dispares. Por ejemplo, si bien la región patagónica y litoraleña reciben en promedio el $48 \%$ de los turistas cada una, la primera abarca el $66 \%$ de la superficie declarada como PN y la segunda solo el $4 \%$.

Figura 10. Arribo de turistas a PN argentinos según región geográfica de localización

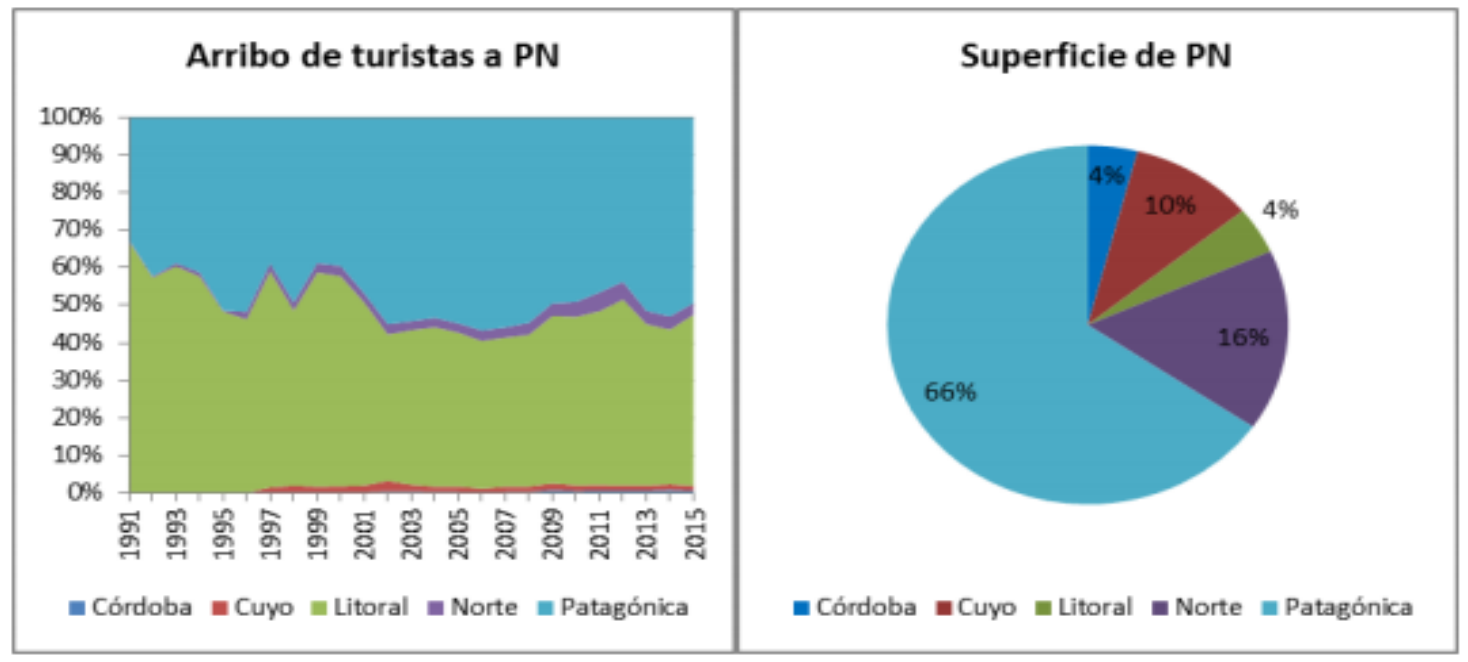

Fuente: Elaboración propia a partir de los Anuarios Estadísticos de Turismo (2007-2015). 
Asimismo, el patrón de crecimiento del arribo de turistas a las distintas regiones de PN también es diverso (Figura 11). En términos generales, se observa como aquellas regiones que cuentan con PN declarados Sitios Patrimoniales presentan tasas de crecimiento anuales mayores durante el segundo subperíodo de análisis. De esta forma, a partir de 2002 el arribo de turistas a estas regiones (Litoral, Norte, Patagonia) crece a una tasa promedio anual del $7 \%$, mientras que la tasa de arribo al resto de las regiones es del $3 \%$.

Figura 11. Arribo de turistas a las regiones argentinas con PN. 1991-2015

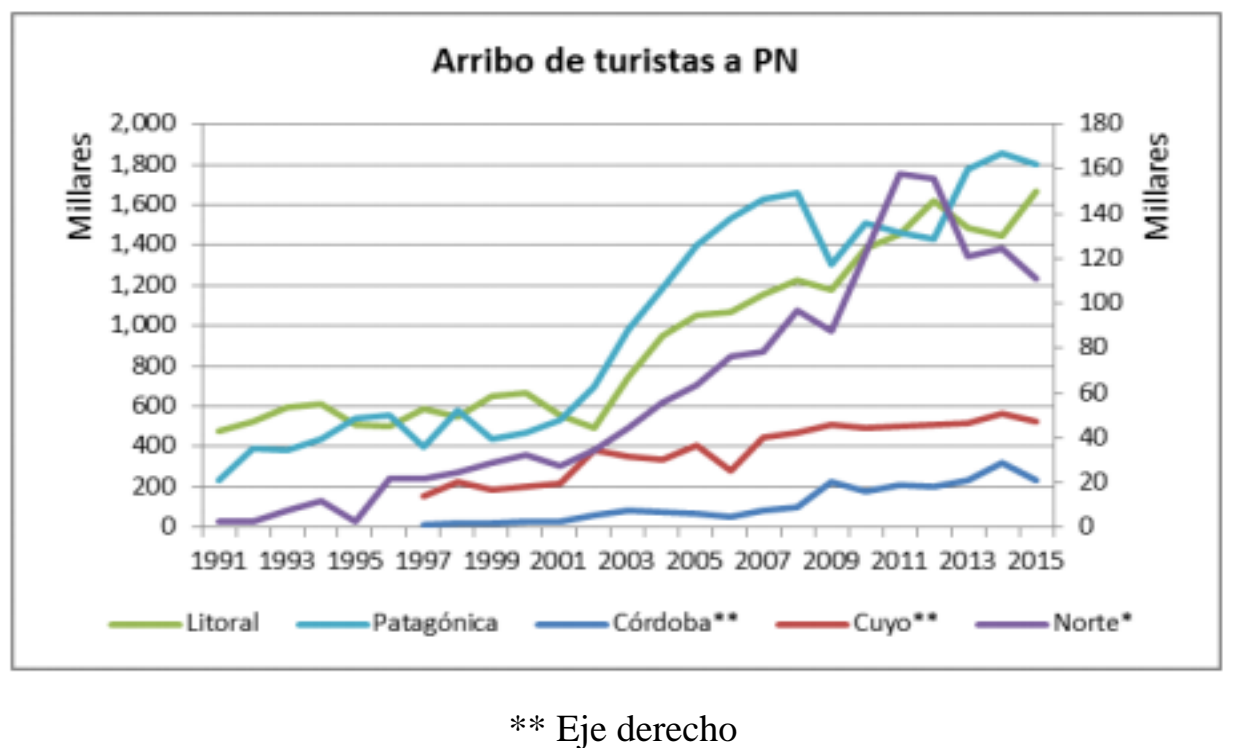

Fuente Elaboración propia a partir de los Anuarios Estadísticos de Turismo (2007-2015)

\subsubsection{Un análisis descriptivo por regiones}

A continuación, se realiza un análisis descriptivo regional del arribo de turistas a PN con el objetivo de observar la concentración del turismo, así como posibles cambios en las preferencias turísticas intrarregionales. En particular se estudian la Patagonia, el Litoral, el Norte y Cuyo. En la región Centro solo se encuentra el PN Quebrada del Condorcito, de modo que este análisis en particular carece de sentido.

En la región patagónica las visitas turísticas se concentran en el PN Nahuel Huapi, 33\% promedio y en el PN Los Glaciares, $25 \%$ promedio (Figura 7). Sin embargo, se aprecian algunas tendencias marcadas que podrían revertir esta situación. Específicamente, se observa cómo el arribo de turistas al PN Nahuel Huapi pierde cada vez más peso relativo pasando de una participación del 70\% en 1991 a una del 17\% en 2015. Lo contrario sucede con el PN Los Glaciares, cuya importancia relativa es cada vez mayor, pasando del $2 \%$ al $41 \%$ en los mismos años. Con participaciones intermedias, les siguen en importancia el PN Tierra del Fuego, 16\%, PN Los Alerces, 9\%; PN Lanín, 8\% y PN Lago Puelo, 6\%. La participación del resto de los PN de la región en el arribo de turistas es menor a la unidad (Figura 12). 
Figura 12. Arribo de turistas al PN de la región patagónica. 1991-2015.

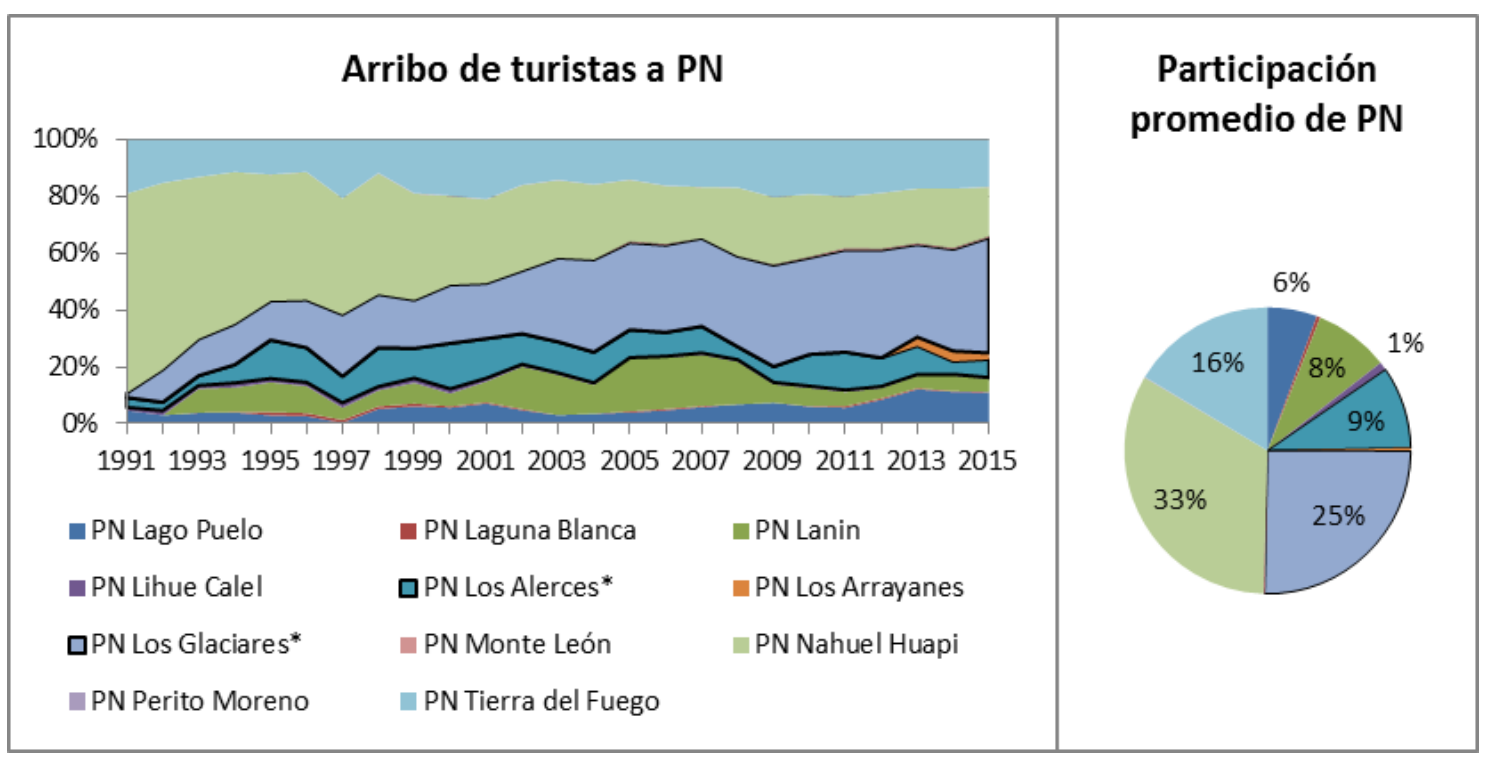

*PN declarado Sitio Patrimonio de la Humanidad (UNESCO, 2019)

Elaboración propia en base a datos de los Anuarios Estadísticos de Turismo (2007-2015).

En el Litoral, como es de esperar, las visitas turísticas a PN se concentran en el PN Iguazú, 86\% en promedio, pasando de una participación del $90 \%$ en 1991 a una del 86\% en 2015, el atractivo de este parque patrimonio de la Humanidad es relativamente estable. Le sigue en importancia el PN El Palmar con una participación promedio del 11\%, aunque creciente. El resto de los parques de la región presentan participaciones cercanas al 1\% (Figura 13).

Figura 13. Arribo de turistas a los PN de la región litoral, 1991-2015.

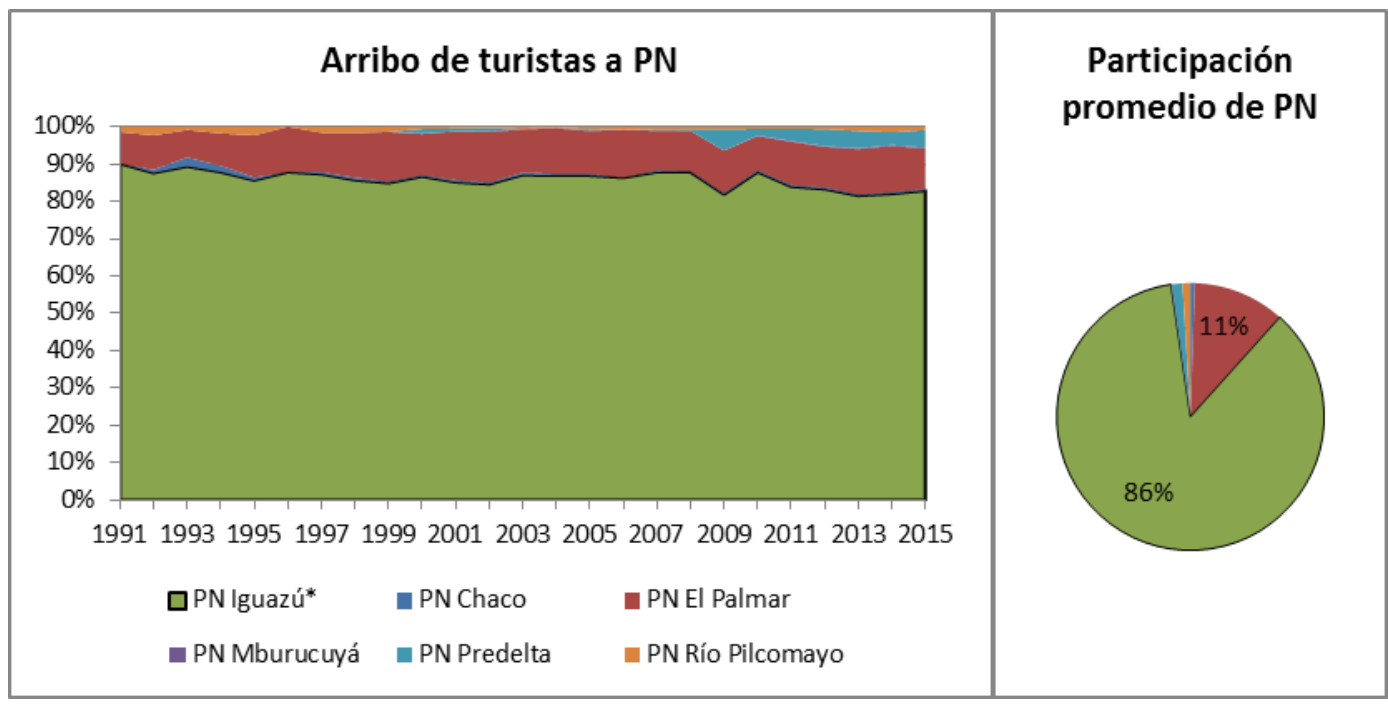

*PN declarado Sitio Patrimonio de la Humanidad (UNESCO, 2019)

Fuente: Elaboración propia a partid de los Anuarios Estadísticos de Turismo (2007-2015). 
En cuanto a los PN del Norte argentino, se observa la preferencia por el PN y Sitio Patrimonial Talamapaya (50\% promedio), seguido del PN Calilegua, con un $30 \%$ de promedio (Figura 14). Sin embargo, esta preferencia dista de ser estática. Al principio del período se observa una clara preferencia por el PN Calilegua con una participación en el turismo de parques de la región que ronda el $90 \%$ hasta mediados de los '90. A partir de entonces se observa que el destino por excelencia pasa a ser el PN Talamapaya con una participación promedio del 70\% hasta 2007. A partir de entonces, si bien este Sitio Patrimonial continúa recibiendo la mayor cantidad de turistas de la región, el PN Los Cardones comienza a ganar atractivo turístico, con una participación promedio para lo que resta del período de casi el $35 \%$ y una participación máxima del 50\% en 2011.

Figura 14. Arribo de turistas a PN de la región norteña. 1991-2015.

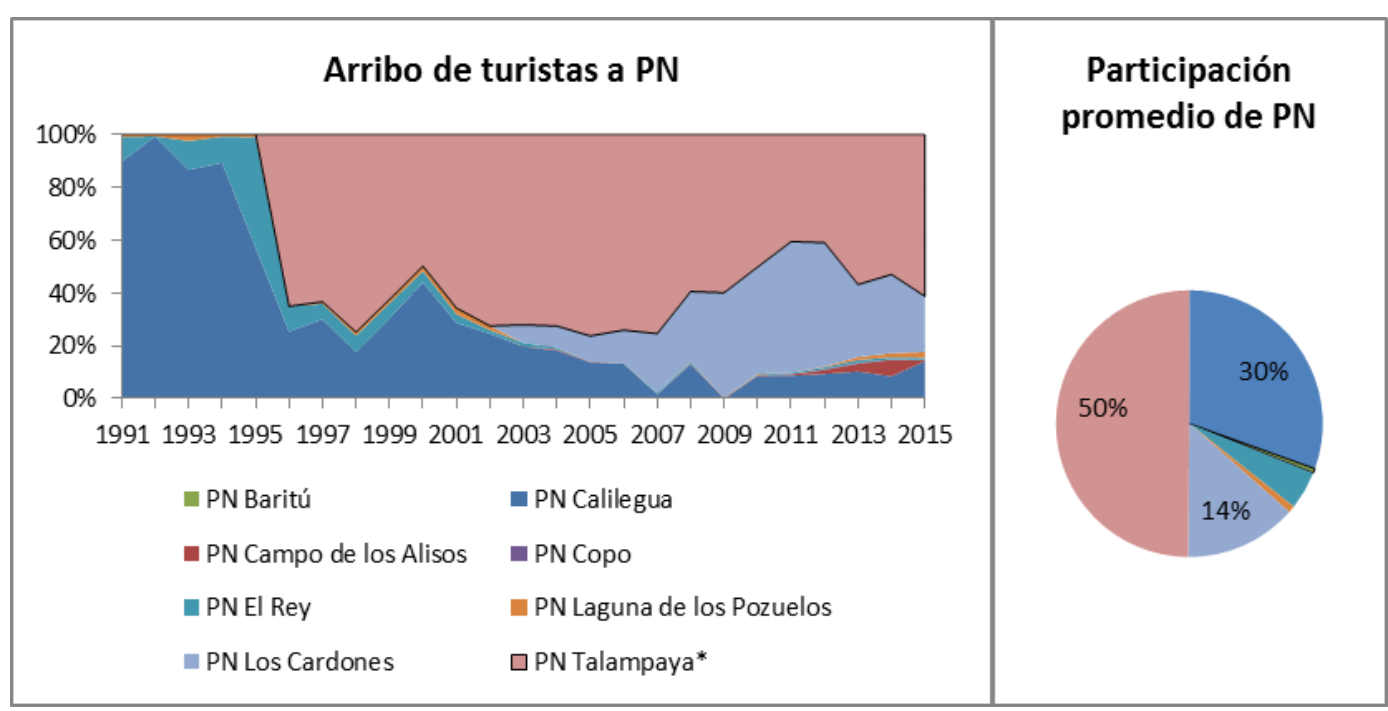

*PN declarado Sitio Patrimonio de la Humanidad (UNESCO, 2019)

Fuente: Elaboración propia a partir de los Anuarios Estadísticos de Turismo (2007-2015).

Finalmente, sin los PN declarados Sitios Patrimoniales, el desarrollo del turismo en el PN de Cuyo es relativamente nuevo, comenzando a mediados de la década de los noventa. El PN Sierra de las Quijadas es el preferido con una participación promedio del 82\%. No obstante, el PN El Leoncito comienza a ganar participación a partir de 2003, llegando a un máximo del $50 \%$ en el final del período (Figura 15). 
Figura 15. Arribo de turistas a PN de la región cuyana. 1991-2015.

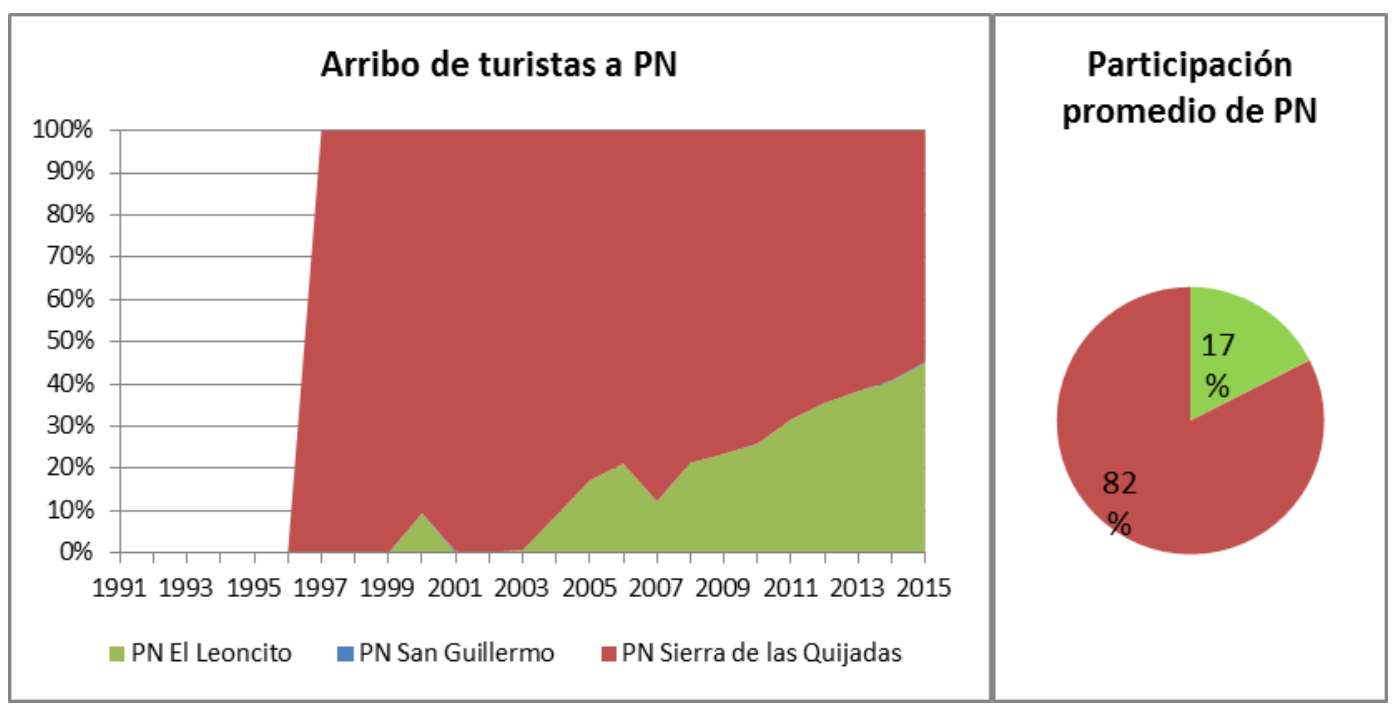

Fuente: Elaboración propia a partir de los Anuarios Estadísticos de Turismo (2007-2015).

\section{Reflexiones finales}

En su vasto territorio, la República Argentina cuenta con variedad de paisajes y patrimonios naturales que a lo largo de su historia han sido declarados Parques Nacionales para mejorar su resguardo y conservación. Varios de ellos incluso han alcanzado el máximo galardón de la UNESCO y han sido declarados Patrimonio Mundial de la Humanidad; lo que ha ayudado a su reconocimiento y apoyo a nivel internacional. En este trabajo se describe la llegada de turistas a los parques nacionales teniendo en cuenta la nominación de la UNESCO como SPM.

Los resultados, si consideramos las cifras globales del total de estos espacios naturales protegidos, muestran que el turismo en los $\mathrm{PN}$ ha crecido exponencialmente durante el período analizado (1991-2015). En términos generales, no obstante, se observa que, a pesar de la diversidad geográfica y patrimonial del territorio argentino, el turismo se concentra abrumadoramente en solo tres de estos parques (Iguazú, Nahuel Huapi y Los Glaciares), de manera que existe una distribución territorial muy desigual de los flujos turísticos a nivel nacional con una macrocefalia destacada de los parques anteriormente mencionados.

El fuerte dinamismo general del turismo en los PN argentinos, se debe al importante crecimiento del arribo de turistas a aquellos PN SPM, sobre todo desde 2001. Pero, en cualquier caso, se observa una marcada preferencia por los PN declarados SPM (Iguazú, Talampaya, Los Alerces y Los Glaciares). Estos resultados parecen confirmar las tesis de diversos autores que encuentran una relación positiva entre el arribo de turistas y las declaraciones oficiales de SPM, que conllevan, en la práctica, una importante promoción y difusión de estos espacios protegidos excepcionales (Ashworth y Turnbridge, 1990; Pocock, 1997; Shackley, 1998).

\section{Referencias}

AET (2007-2015). Anuario Estadístico de Turismo. Ministerio de Turismo. Años 2007, 2008, 2009, 2010, 2011, 2012, 2013, 2014 y 2015. Buenos Aires: Presidencia de la Nación.

Ashworth, G. y Turnbridge, J. (1990). The Tourist Historic City. London: Belhaven. 
Berjman, S. y Gutiérrez, R. (1985). Patrimonio cultural y patrimonio natural: la arquitectura en los parques nacionales Nahuel Huapi e Iguazú hasta 1950. Buenos Aires: Instituto Argentino de Investigaciones de Historia de la Arquitectura y del Urbanismo.

Consultora Elypsis (2018). Informe "Turismo de Naturaleza" elaborado a pedido de la Fundación Flora y Fauna. Buenos Aires: Elypsis, informe inédito.

Galvin, D. (1997). Testimony on H.R. 901, The American Land Sovereignty Protection Act, US. 105th Congress House of Representatives Committee on Resources. Washington DC: ALSPA.

Elías, L. y Tortul, E. (2018). Sitios Patrimonio de la Humanidad y Turismo: Una aproximación a su impacto en América Latina y Caribe. En LIII Reunión Anual de la Asociación Argentina de Economía Política. La Plata, Argentina: Asociación Argentina de Economía Política.

Mulero Mendigorri, A. y Rivera Mateos, M. (2018). Turismo de naturaleza y espacios naturales protegidos en España. Abaco: Revista de cultura y ciencias sociales, 98 (Ejemplar dedicado a: El turismo. Desarrollo, transformación y controversia de un fenómeno social), 84-96

Piglia, M. (2012). En torno a los Parques Nacionales: primeras experiencias de una política turística nacional centralizada en la Argentina (1934-1950) PASOS. Revista de Turismo y Patrimonio Cultural, 10 (1), 61-73.

Pocock, D. (1997). Some Reflections on World Heritage, Area, 29(3), 260-268.

Shackley, M. (1998). Visitor Management: Case Studies from World Heritage Sites. Oxford: Butterworth-Heinemann.

Silva Pérez, R., \& Fernández Salinas, V. (2015). Los paisajes culturales de Unesco desde la perspectiva de América Latina y el Caribe: Conceptualizaciones, situaciones y potencialidades. Revista Invi, 30(85), 181-214.

UNESCO (2000). El Patrimonio de la Humanidad. Madrid: Planeta de Agostini.

UNESCO (2019). Lista de Sitios Patrimoniales Mundiales. https://whc.unesco.org/en/list/ 